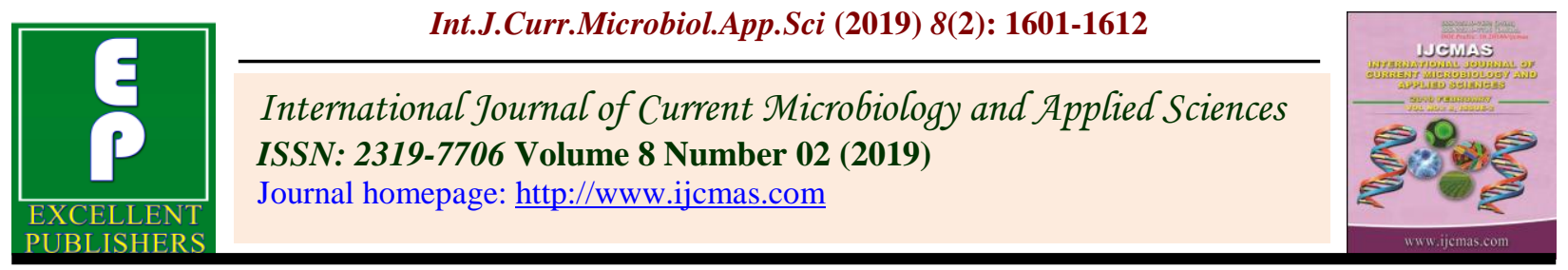

\title{
Study on Association of Bio-physiological Parameters with Grain Yield in Sorghum Genotypes under Post Flowering Moisture Stress Conditions
}

\author{
D. Devkumar ${ }^{1 *}$, V. Padma ${ }^{2}$, H.S. Talwar ${ }^{3}$ and Farzana Jabeen ${ }^{4}$ \\ ${ }^{1}$ Department of Crop Physiology, College of Agriculture, Prof. Jayashankar Telangana State \\ Agricultural University (PTSAU), Rajendranagar, Hyderabad-30, India \\ ${ }^{2}$ Department of Crop Physiology, Lam form Guntur, India \\ ${ }^{3}$ Department of Plant Physiology, Indian institute of Millet Research (IIMR), Rajendranagar, \\ Hyderabad -30, India \\ ${ }^{4}$ Department of Genetics and Plant Breeding, College of Agriculture, Prof. Jayashankar \\ Telangana State Agricultural University (PTSAU), Rajendranagar, Hyderabad-30, India \\ *Corresponding author
}

\section{A B S T R A C T}

Keywords

SPAD Clorophyll

Meter Reading

(SCMR),

Photosynthetic rate,

Transpiration rate,

Stomatal resistance,

Grain yield,

Sorghum genotypes

Article Info

Accepted:

10 January 2019

Available Online:

10 February 2019

\begin{abstract}
A field experiment was conducted during rabi 2012-13 at research farm of Directorate of Sorghum Research, Rajendranagar, Hyderabad. The experiment was laid out in a split plot design, replicated thrice, with 10 Sorghum genotypes as main treatment (well watered and water stress conditions) and with 10 genotypes are sub treatments CRS 4, CRS 19, CRS 20, PEC 17, CSV 18, M 35-1, Phule chitra, Phule moulee, EP 57 and CRS 1). Photosynthetic rate and stomatal resistance at 15 and 30 DAF were positively and significantly correlated with grain yield while the transpiration rate at 15 and 30 DAF exhibited negative correlation with grain yield. SPAD chlorophyll meter reading (15 and $30 \mathrm{DAF}$ ) and chlorophyll content at $30 \mathrm{DAF}$ had positively significant correlation with grain yield.
\end{abstract}

\section{Introduction}

Sorghum (Sorghum bicolor (L.) Moench) is one of the world's most important nutritional cereal crops and also the major staple food crop of millions of people in semi-arid tropics (SAT). It is considered as the king of millets and extensively grown in Africa, China, USA, Mexico and India. Sorghum ranks fourth among the world's most important crops after wheat, rice and maize. Its current world production stands at 64.6 million tonnes while in India current production is 7.4 million tonnes. In India, Sorghum is cultivated in both rainy and post rainy (rabi) season, mainly as a rain fed crop with about $85 \%$ of the production concentrated in Maharashtra, Karnataka and Andhra Pradesh. The national average productivity of Sorghum is very low (880 kg/ha). In India, it is the major dry land 
crop currently grown in about $7.69 \mathrm{~m}$ ha during both kharif (3.2 $\mathrm{m} \mathrm{ha})$ and rabi (4.50 $\mathrm{m} \mathrm{ha}$ ) seasons with a production of $7.73 \mathrm{~m} \mathrm{t}$.

The rabi Sorghum is normally grown under stored and receding soil moisture conditions with increasing temperature after flowering. Thus, it experiences both soil and atmospheric water deficit (drought). The limited availability of water causes moisture stress which affects various metabolic processes of the plant. The limited availability of water causes moisture stress which affects various metabolic processes of the plant. The major limitations for Sorghum productivity are the occurrence of various biotic (shoot fly, stem borer, charcoal rot etc) and abiotic (drought, salinity and temperature, etc.) stresses at different crop growth stages.

\section{Materials and Methods}

The treatments comprised to screen the promising germplasm, advanced breeding lines and landraces to identify the new sources and traits associated with post flowering drought tolerance in sorghum. The crop was sown under well watered and water stress condition to examine the potential of Sorghum genotypes to adapt to the post flowering drought. Well Watered and Water Stress (two main treatments) conditions and 10 Sorghum genotypes viz; CRS 4, CRS 19, CRS 20, PEC 17, CSV 18, M 35-1, Phule chitra, Phule moulee, EP 57 and CRS 1. The experiment was laid out in split plot design and replicated thrice. The SPAD-502 (Soil Plant Analytical Development) meter was used for measuring the relative chlorophyll content of leaves. The readings were taken from top third fully expanded leaf. Mean of five values from five hills was obtained. The photosynthetic rate, transpiration rate and stomatal resistance were measured in the $3^{\text {rd }}$ fully expanded leaf from the top by using Infra Red Gas Analyzer (Model TPS-1). The data on were analyzed statistically by applying the technique of split plot design taken from (Panse and Sukhatme, 1978).

The spacing maintained was $60 \mathrm{~cm}$ between rows and $15 \mathrm{~cm}$ between plants. A basal dose of $20 \mathrm{~kg} \mathrm{ha}^{-1} \mathrm{~N}$ and $20 \mathrm{~kg} \mathrm{ha}{ }^{-1} \mathrm{P}_{2} \mathrm{O}_{5}$ was applied before final ploughing. The seed were hand sown and the field was irrigated to saturate the soil profile with water to ensure uniform germination. The crop was thinned to two plants per hill after 10 days of emergence and then to one plant per hill after about a week. Around 20 days after emergence, an additional $20 \mathrm{~kg} \mathrm{ha}^{-1} \mathrm{~N}$ as urea was applied and irrigated.

\section{Results and Discussion}

\section{SPAD Chlorophyll Meter Reading (SCMR)}

The data on SPAD reading revealed significant differences among the genotypes both at 15 and 30 DAF and the maximum SPAD readings was recorded at 15 DAF by all the genotypes compared to $30 \mathrm{DAF}$ are presented in table 1 and figure 1.

At 15 DAF, the genotype PEC 17 (51) had the maximum SPAD reading and the lowest SPAD reading was CRS 1 (38). At 30 DAF the maximum SPAD readings was recorded in PEC 17 (37). The lowest SPAD reading at this stage was recorded in the genotype CRS 1 (24). Significant differences were also observed between the treatments, during well watered and water stress conditions. The SPAD readings decreased in all the genotypes due to the moisture stress imposed during post flowering period. The SPAD chlorophyll meter readings had significant and positive correlation with grain yield both at $15 \mathrm{DAF}$ ( $\mathrm{r}$ $=0.80)$ and 30 DAF $(r=0.50)$.

So, SCMR can be used to evaluate the performance of Sorghum genotypes under 
post flowering drought condition. In general, higher SCMR means greater nitrogen and chlorophyll and thus these values can be taken as an index for evaluation of Sorghum genotypes for drought tolerance. The results observed in the present study are in conformity with the results of $\mathrm{Xu}$ et al., (2000) Rao et al., (2003) (Talwar et al., 2011) and Sudhakar et al., (2006).

\section{Photosynthetic rate $\left(\mu \mathrm{mol} \mathrm{CO}_{2} \mathrm{~m}^{-2} \mathrm{~s}^{-1}\right)$}

The data on photosynthetic rate revealed significant differences among the genotypes both at 15 and 30 DAF and the maximum photosynthetic rate was recorded at $15 \mathrm{DAF}$ by all the genotypes compared to $30 \mathrm{DAF}$ (Table 2 and Fig. 2).

At 15 DAF, the genotype PEC $17(36.5 \mu \mathrm{mol}$ $\mathrm{CO}^{2} \mathrm{~m}^{2} \mathrm{~s}^{-1}$ ) had the maximum photosynthetic rate followed by $\mathrm{M}$ 35-1 $\left(35.5 \mu \mathrm{mol} \mathrm{CO}^{2} \mathrm{~m}^{2}\right.$ $\left.\mathrm{s}^{-1}\right)$ and CSV $18\left(32.5 \mu \mathrm{mol} \mathrm{CO} \mathrm{CO}^{2} \mathrm{~s}^{-1}\right)$, and the lowest photosynthetic rate was in CRS 1 (25.5 $\left.\mu \mathrm{mol} \mathrm{CO}^{2} \mathrm{~m}^{2} \mathrm{~s}^{-1}\right)$. At 30 DAF the maximum photosynthetic rate was recorded in PEC 17 (26.5 $\left.\mu \mathrm{mol} \mathrm{CO} \mathrm{CO}^{2} \mathrm{~s}^{-1}\right)$ followed by M 35-1 (25.5 $\left.\mu \mathrm{mol} \mathrm{CO} \mathrm{CO}^{2} \mathrm{~s}^{-1}\right)$.

The lowest photosynthetic rate at this stage was recorded by the same genotype CRS 1 (16.5 $\left.\mu \mathrm{mol} \mathrm{CO}^{2} \mathrm{~m}^{2} \mathrm{~s}^{-1}\right)$. Such variation in photosynthetic rate among genotypes was also reported by Watling et al., (2003), Rao et al., (2001), Pawar et al., (2005) and Channappagoudar et al., (2008). There was significant difference between the treatments, during well watered and water stress. The photosynthetic rate decreased in all the genotypes due to the moisture stress impose during post flowering period. The photosynthetic rate was positively and significantly correlated with grain yield at 15DAF $(r=0.71)$ and $30 \mathrm{DAF}(\mathrm{r}=0.57)$.

\section{Transpiration rate $\left(\mu \mathrm{mol} \mathrm{H}_{2} \mathrm{O} \mathrm{m}^{2} \mathrm{~s}^{-1}\right)$}

The data on transpiration rate revealed significant differences among the genotypes at 15 and 30 DAF and the maximum transpiration rate was recorded at $15 \mathrm{DAF}$ compared to 30 DAF (Table 3 and Fig. 3).

At 15 DAF, maximum transpiration rate was recorded in CRS $1\left(4.28 \mu \mathrm{mol} \mathrm{H}_{2} \mathrm{O} \mathrm{m}^{2} \mathrm{~s}^{-1}\right)$ followed by CRS $20\left(4.17 \mu \mathrm{mol} \mathrm{H}_{2} \mathrm{O} \mathrm{m}^{2} \mathrm{~s}^{-1}\right)$. The lowest transpiration rate at this stage was recorded in the genotype PEC $17(2.43 \mu \mathrm{mol}$ $\left.\mathrm{H}_{2} \mathrm{O} \mathrm{m}^{2} \mathrm{~s}^{-1}\right)$ and $\mathrm{M} \mathrm{35-1}\left(2.69 \mu \mathrm{mol} \mathrm{H} \mathrm{O} \mathrm{m}^{2}\right.$ $\left.\mathrm{s}^{-1}\right)$. Similarly, at $30 \mathrm{DAF}$, the maximum transpiration rate was recorded in CRS 1 $\left(2.70 \mu \mathrm{mol} \mathrm{H}_{2} \mathrm{O} \mathrm{m}^{2} \mathrm{~s}^{-1}\right)$ followed by CRS 20 $\left(2.58 \mu \mathrm{mol} \mathrm{H}_{2} \mathrm{O} \mathrm{m}^{2} \mathrm{~s}^{-1}\right)$. The lowest transpiration rate was recorded in PEC 17 $\left(0.87 \mu \mathrm{mol} \mathrm{H}_{2} \mathrm{O} \mathrm{m}^{2} \mathrm{~s}^{-1}\right)$ and $\mathrm{M} \mathrm{35-1}(0.96 \mu$ mol $\left.\mathrm{H}_{2} \mathrm{O} \mathrm{m} \mathrm{m}^{2} \mathrm{~s}^{-1}\right)$. Similarly, the genotypic variations in transpiration rate were also reported by several workers (Dhopte et al., 1987 and Yadav et al., 1991). Significant differences were also observed between the treatments, during well watered and water stress conditions. There was increase in transpiration rate in all the genotypes due to the moisture stress induced during post flowering period. Higher transpiration efficiency was desirable for higher grain yield and biomass productivity under post anthesis drought stress situations was earlier reported by Rao et al., 2001. The transpiration rate was negatively and significantly correlated with grain yield both at $15 \mathrm{DAF}(\mathrm{r}=-0.54)$ and 30 DAF $(r=0.56)$ (Table 5).

Under receding soil moisture situation, maintenance of low transpiration rate is an important factor for yield stability. The lower transpiration rate as a trait can be incorporated into the hybrids for better yields under receding soil moisture situation (Ashok Surveshi et al., 2011). 
Table.1 SPAD readings at 15 DAF and 30 DAF of Sorghum genotypes under well watered and water stress conditions

\begin{tabular}{|c|c|c|c|c|c|c|}
\hline \multirow[b]{2}{*}{ Genotypes } & \multicolumn{3}{|c|}{ SPAD - 15DAF } & \multicolumn{3}{|c|}{ SPAD - 30DAF } \\
\hline & WW & WS & Mean & WW & WS & Mean \\
\hline CRS 4 & 50 & 45 & 48 & 32 & 28 & 30 \\
\hline CRS 19 & 44 & 40 & 42 & 33 & 30 & 32 \\
\hline CRS 20 & 48 & 43 & 46 & 27 & 25 & 26 \\
\hline PEC 17 & 52 & 49 & 51 & 38 & 35 & 37 \\
\hline CSV 18 & 46 & 45 & 46 & 32 & 32 & 32 \\
\hline M35-1 & 49 & 45 & 47 & 34 & 31 & 33 \\
\hline Phule Chitra & 45 & 43 & 44 & 32 & 29 & 31 \\
\hline Phule Moulee & 45 & 41 & 43 & 37 & 34 & 36 \\
\hline EP 57 & 46 & 42 & 44 & 33 & 31 & 32 \\
\hline CRS 1 & 39 & 37 & 38 & 24 & 23 & 24 \\
\hline Mean & 46 & 43 & 45 & 32 & 30 & 31 \\
\hline $\begin{array}{l}\text { CD } \\
\text { Genotypes (G) }\end{array}$ & \multicolumn{3}{|c|}{4.63} & \multicolumn{3}{|c|}{4.23} \\
\hline Treatments $(\mathrm{T})$ & \multicolumn{3}{|c|}{2.25} & \multicolumn{3}{|c|}{2.23} \\
\hline G X T & \multicolumn{3}{|c|}{7.12} & \multicolumn{3}{|c|}{7.06} \\
\hline CV & \multicolumn{3}{|c|}{9.33} & \multicolumn{3}{|c|}{13.37} \\
\hline
\end{tabular}

WW-Well Watered, WS- Water Stress.

Table.2 Photosynthetic rate $\left(\mu \mathrm{mol} \mathrm{CO} \mathrm{m}^{2} \mathrm{~s}^{-1}\right)$ at $15 \mathrm{DAF}$ and $30 \mathrm{DAF}$ of Sorghum genotypes under well watered and water stress conditions

\begin{tabular}{|c|c|c|c|c|c|c|}
\hline \multirow[b]{2}{*}{ Genotypes } & \multicolumn{3}{|c|}{ Photosynthetic rate - 15DAF } & \multicolumn{3}{|c|}{ Photosynthetic rate - 30DAF } \\
\hline & WW & WS & Mean & WW & WS & Mean \\
\hline CRS 4 & 31 & 30 & 30.5 & 20 & 19 & 19.5 \\
\hline CRS 19 & 30 & 27 & 28.5 & 20 & 20 & 20.0 \\
\hline CRS 20 & 27 & 26 & 26.5 & 18 & 17 & 17.5 \\
\hline PEC 17 & 37 & 36 & 36.5 & 27 & 26 & 26.5 \\
\hline CSV 18 & 33 & 32 & 32.5 & 21 & 20 & 20.5 \\
\hline M35-1 & 36 & 35 & 35.5 & 26 & 25 & 25.5 \\
\hline Phule Chitra & 27 & 26 & 26.5 & 18 & 16 & 17.0 \\
\hline Phule Moulee & 28 & 26 & 27.0 & 19 & 17 & 18.0 \\
\hline EP 57 & 33 & 31 & 32.0 & 22 & 20 & 21.0 \\
\hline CRS 1 & 26 & 25 & 25.5 & 17 & 16 & 16.0 \\
\hline Mean & 31 & 30 & 30.5 & 21 & 20 & 20.5 \\
\hline $\begin{array}{l}\text { CD } \\
\text { Genotypes (G) }\end{array}$ & \multicolumn{3}{|c|}{2.22} & \multicolumn{3}{|c|}{1.67} \\
\hline Treatments $(\mathbf{T})$ & \multicolumn{3}{|c|}{0.84} & \multicolumn{3}{|c|}{0.84} \\
\hline G X T & \multicolumn{3}{|c|}{2.66} & \multicolumn{3}{|c|}{2.66} \\
\hline CV & \multicolumn{3}{|c|}{7.77} & \multicolumn{3}{|c|}{3.26} \\
\hline
\end{tabular}

WW-Well Watered, WS- Water Stress. 
Table.3 Transpiration rate $\left(\mu \mathrm{mol} \mathrm{H}_{2} \mathrm{O} \mathrm{m}^{2} \mathrm{~s}^{-1}\right)$ at $15 \mathrm{DAF}$ and 30 DAF of Sorghum genotypes under well watered and water stress conditions

\begin{tabular}{|c|c|c|c|c|c|c|}
\hline \multirow[b]{2}{*}{ Genotypes } & \multicolumn{3}{|c|}{ Transpiration rate -15DAF } & \multicolumn{3}{|c|}{ Transpiration rate -30DAF } \\
\hline & WW & WS & Mean & WW & WS & Mean \\
\hline CRS 4 & 3.40 & 3.73 & 3.56 & 2.27 & 2.42 & 2.35 \\
\hline CRS 19 & 3.20 & 3.34 & 3.27 & 2.07 & 2.11 & 2.09 \\
\hline CRS 20 & 4.15 & 4.18 & 4.17 & 2.53 & 2.63 & 2.58 \\
\hline PEC 17 & 2.32 & 2.53 & 2.43 & 0.84 & 0.89 & 0.87 \\
\hline CSV 18 & 2.93 & 3.10 & 3.02 & 1.14 & 1.17 & 1.16 \\
\hline M35-1 & 2.54 & 2.84 & 2.69 & 0.91 & 1.00 & 0.96 \\
\hline Phule Chitra & 3.57 & 3.74 & 3.66 & 2.33 & 2.40 & 2.37 \\
\hline Phule Moulee & 4.01 & 4.06 & 4.04 & 2.64 & 2.72 & 2.68 \\
\hline EP 57 & 3.05 & 3.12 & 3.09 & 1.19 & 1.24 & 1.22 \\
\hline CRS 1 & 4.23 & 4.33 & 4.28 & 2.65 & 2.74 & 2.70 \\
\hline Mean & 3.34 & 3.50 & 3.42 & 1.86 & 1.93 & 1.90 \\
\hline $\begin{array}{l}\text { CD } \\
\text { Genotypes (G) }\end{array}$ & \multicolumn{3}{|c|}{0.14} & \multicolumn{3}{|c|}{0.08} \\
\hline Treatments (T) & \multicolumn{3}{|c|}{0.06} & \multicolumn{3}{|c|}{0.06} \\
\hline G X T & \multicolumn{3}{|c|}{0.19} & \multicolumn{3}{|c|}{0.19} \\
\hline CV & \multicolumn{3}{|c|}{3.26} & \multicolumn{3}{|c|}{6.08} \\
\hline
\end{tabular}

WW-Well Watered, WS- Water Stress

Table.4 Stomata resistance $\left(\mathrm{s} . \mathrm{cm}^{-1}\right)$ at $15 \mathrm{DAF}$ and 30 DAF of Sorghum genotypes under well watered and water stress conditions

\begin{tabular}{|c|c|c|c|c|c|c|}
\hline \multirow[b]{2}{*}{ Genotypes } & \multicolumn{3}{|c|}{ Stomatal resistance $-15 \mathrm{DAF}$} & \multicolumn{3}{|c|}{ Stomatal resistance $-30 D A F$} \\
\hline & WW & WS & Mean & WW & WS & Mean \\
\hline CRS 4 & 45 & 44 & 44.5 & 29 & 28 & 28.5 \\
\hline CRS 19 & 42 & 41 & 41.5 & 26 & 25 & 25.5 \\
\hline CRS 20 & 35 & 34 & 34.5 & 23 & 22 & 22.5 \\
\hline PEC 17 & 54 & 53 & 53.5 & 37 & 36 & 36.5 \\
\hline CSV 18 & 47 & 46 & 46.5 & 31 & 30 & 30.5 \\
\hline M35-1 & 52 & 51 & 51.5 & 33 & 33 & 33.0 \\
\hline Phule Chitra & 43 & 42 & 42.5 & 26 & 25 & 25.5 \\
\hline Phule Moulee & 38 & 37 & 37.5 & 28 & 27 & 27.5 \\
\hline EP 57 & 50 & 49 & 49.5 & 31 & 30 & 30.5 \\
\hline CRS 1 & 36 & 34 & 34.5 & 25 & 24 & 24.5 \\
\hline Mean & 44 & 43 & 43.5 & 29 & 28 & 28.5 \\
\hline $\begin{array}{l}\text { CD } \\
\text { Genotypes (G) }\end{array}$ & \multicolumn{3}{|c|}{1.10} & \multicolumn{3}{|c|}{1.38} \\
\hline Treatments (T) & \multicolumn{3}{|c|}{0.63} & \multicolumn{3}{|c|}{0.61} \\
\hline G X T & \multicolumn{3}{|c|}{2.00} & \multicolumn{3}{|c|}{1.95} \\
\hline CV & \multicolumn{3}{|c|}{2.69} & \multicolumn{3}{|c|}{4.03} \\
\hline
\end{tabular}


Table.5 Correlation Coefficient among fifteen yield and yield related attributes in 10 genotypes of Sorghum

\begin{tabular}{|c|c|c|c|c|c|c|c|c|c|c|c|c|}
\hline Characters & PH & GLAR10 & GLAR 20 & GLAR 30 & GLAR 40 & PSR 15 & PSR 30 & TRAS 15 & TRAS 30 & $\begin{array}{c}\text { STOM } \\
15\end{array}$ & STOM 30 & SPAD 15 \\
\hline PH & 1.00000 & 0.74955 & 0.56558 & 0.74505 & 0.76535 & 0.45749 & 0.31157 & -0.52112 & -0.38139 & 0.46524 & 0.40532 & 0.72213 \\
\hline GLAR 10 & & 1.00000 & 0.76666 & 0.75924 & 0.81673 & 0.66750 & 0.57251 & -0.68575 & -0.62819 & 0.60310 & 0.56893 & 0.79546 \\
\hline GLAR 20 & & & 1.00000 & 0.78614 & 0.78170 & 0.47241 & 0.48748 & -0.59095 & -0.55425 & 0.46550 & 0.34905 & 0.52267 \\
\hline GLAR 30 & & & & 1.00000 & 0.92397 & 0.37803 & 0.36612 & -0.50432 & -0.35114 & 0.36082 & 0.25450 & 0.56451 \\
\hline GLAR 40 & & & & & 1.00000 & 0.44195 & 0.39801 & -0.53670 & -0.41178 & 0.41128 & 0.36596 & 0.69483 \\
\hline PSR 15 & & & & & & 1.00000 & 0.93217 & -0.93434 & -0.92832 & 0.93381 & 0.94319 & 0.73645 \\
\hline PSR 30 & & & & & & & 1.00000 & -0.90752 & -0.87254 & 0.86114 & 0.88265 & 0.61612 \\
\hline TRAS 15 & & & & & & & & 1.00000 & 0.94382 & -0.95700 & -0.88733 & -0.64545 \\
\hline TRAS 30 & & & & & & & & & 1.00000 & -0.92537 & -0.87505 & -0.55245 \\
\hline STOM 15 & & & & & & & & & & 1.00000 & 0.92285 & 0.64516 \\
\hline STOM 30 & & & & & & & & & & & 1.00000 & 0.65296 \\
\hline SPAD 15 & & & & & & & & & & & & 1.00000 \\
\hline
\end{tabular}

\begin{tabular}{|c|c|c|c|c|c|c|c|c|c|c|c|}
\hline Charecters & SPAD 30 & $\begin{array}{l}\text { Chlorophyll } \\
\text { content }\end{array}$ & $\mathrm{N}$ conent & K conent & $\begin{array}{l}\text { Panicle } \\
\text { length }\end{array}$ & $\begin{array}{c}\text { Panicle } \\
\text { weight }\end{array}$ & $\begin{array}{c}1000 \\
\text { seed wt }\end{array}$ & GWP & $\begin{array}{c}\text { No } \\
\text { Grains } \\
\text { per } \\
\text { panicle }\end{array}$ & HI & GY \\
\hline PH & 0.48181 & 0.40671 & 0.21529 & 0.23607 & 0.49609 & 0.53381 & 0.34717 & 0.49170 & 0.69721 & 0.59234 & 0.50869 \\
\hline GLAR 10 & 0.60293 & 0.61462 & 0.54715 & 0.47557 & 0.53517 & 0.43580 & 0.50709 & 0.53641 & 0.64479 & 0.67829 & 0.66093 \\
\hline GLAR 20 & 0.40050 & 0.48520 & 0.43800 & 0.34156 & 0.25355 & 0.68761 & 0.44809 & 0.59485 & 0.62873 & 0.55128 & 0.27237 \\
\hline GLAR 30 & 0.43024 & 0.32470 & 0.25703 & 0.26102 & 0.27778 & 0.60753 & 0.34445 & 0.57896 & 0.74304 & 0.56010 & 0.30798 \\
\hline GLAR 40 & 0.58200 & 0.44763 & 0.30556 & 0.31126 & 0.39531 & 0.61981 & 0.50042 & 0.66013 & 0.67872 & 0.68520 & 0.47684 \\
\hline PSR 15 & 0.63724 & 0.94841 & 0.90911 & 0.86078 & 0.78182 & 0.29867 & 0.56543 & 0.38855 & 0.40838 & 0.67004 & 0.71594 \\
\hline PSR 30 & 0.57334 & 0.89585 & 0.90141 & 0.91585 & 0.64511 & 0.30617 & 0.51505 & 0.42145 & 0.41373 & 0.69083 & 0.57892 \\
\hline TRAS 15 & -0.67680 & -0.91599 & -0.83443 & -0.78232 & -0.67073 & -0.49802 & -0.57436 & -0.51755 & -0.44591 & -0.65210 & -0.53955 \\
\hline TRAS 30 & -0.54711 & -0.92177 & -0.86935 & -0.72392 & -0.62204 & -0.45570 & -0.55184 & -0.42222 & -0.27355 & -0.60292 & -0.56452 \\
\hline STOM 15 & 0.64676 & 0.92555 & 0.79782 & 0.77433 & 0.65443 & 0.33882 & 0.47695 & 0.32245 & 0.31607 & 0.59949 & 0.56848 \\
\hline STOM 30 & 0.72119 & 0.96940 & 0.86006 & 0.87122 & 0.74686 & 0.22516 & 0.61112 & 0.37231 & 0.27032 & 0.66100 & 0.69925 \\
\hline SPAD 15 & 0.63797 & 0.67609 & 0.55359 & 0.59953 & 0.78576 & 0.30853 & 0.50596 & 0.43915 & 0.68566 & 0.71577 & 0.80475 \\
\hline SPAD 30 & 1.00000 & 0.73445 & 0.55918 & 0.60357 & 0.62288 & 0.32374 & 0.82722 & 0.65336 & 0.35218 & 0.57278 & 0.50430 \\
\hline Chloro & & 1.00000 & 0.90815 & 0.86886 & 0.74944 & 0.37629 & 0.69990 & 0.48934 & 0.33893 & 0.65170 & 0.65086 \\
\hline $\mathrm{N}$ content & & & 1.00000 & 0.91038 & 0.71761 & 0.30344 & 0.63435 & 0.45291 & 0.38183 & 0.45255 & 0.51861 \\
\hline$K$ content & & & & 1.00000 & 0.67531 & 0.15494 & 0.52818 & 0.36415 & 0.42784 & 0.51902 & 0.51316 \\
\hline Panicle length & & & & & 1.00000 & 0.34468 & 0.68815 & 0.53798 & 0.55946 & 0.48976 & 0.72169 \\
\hline Pl wt & & & & & & 1.00000 & 0.54386 & 0.82000 & 0.59558 & 0.35277 & 0.03855 \\
\hline 1000 seed wt & & & & & & & 1.00000 & 0.84791 & 0.38413 & 0.46841 & 0.44820 \\
\hline GWP & & & & & & & & 1.00000 & 0.63354 & 0.45358 & 0.22574 \\
\hline $\begin{array}{l}\text { No of grains per } \\
\text { panicle }\end{array}$ & & & & & & & & & 1.00000 & 0.39835 & 0.28708 \\
\hline HI & & & & & & & & & & 1.00000 & 0.78341 \\
\hline GY & & & & & & & & & & & 1.00000 \\
\hline
\end{tabular}


Fig.1 SPAD readings at 15 DAF and 30 DAF of Sorghum genotypes under well watered and water stress conditions

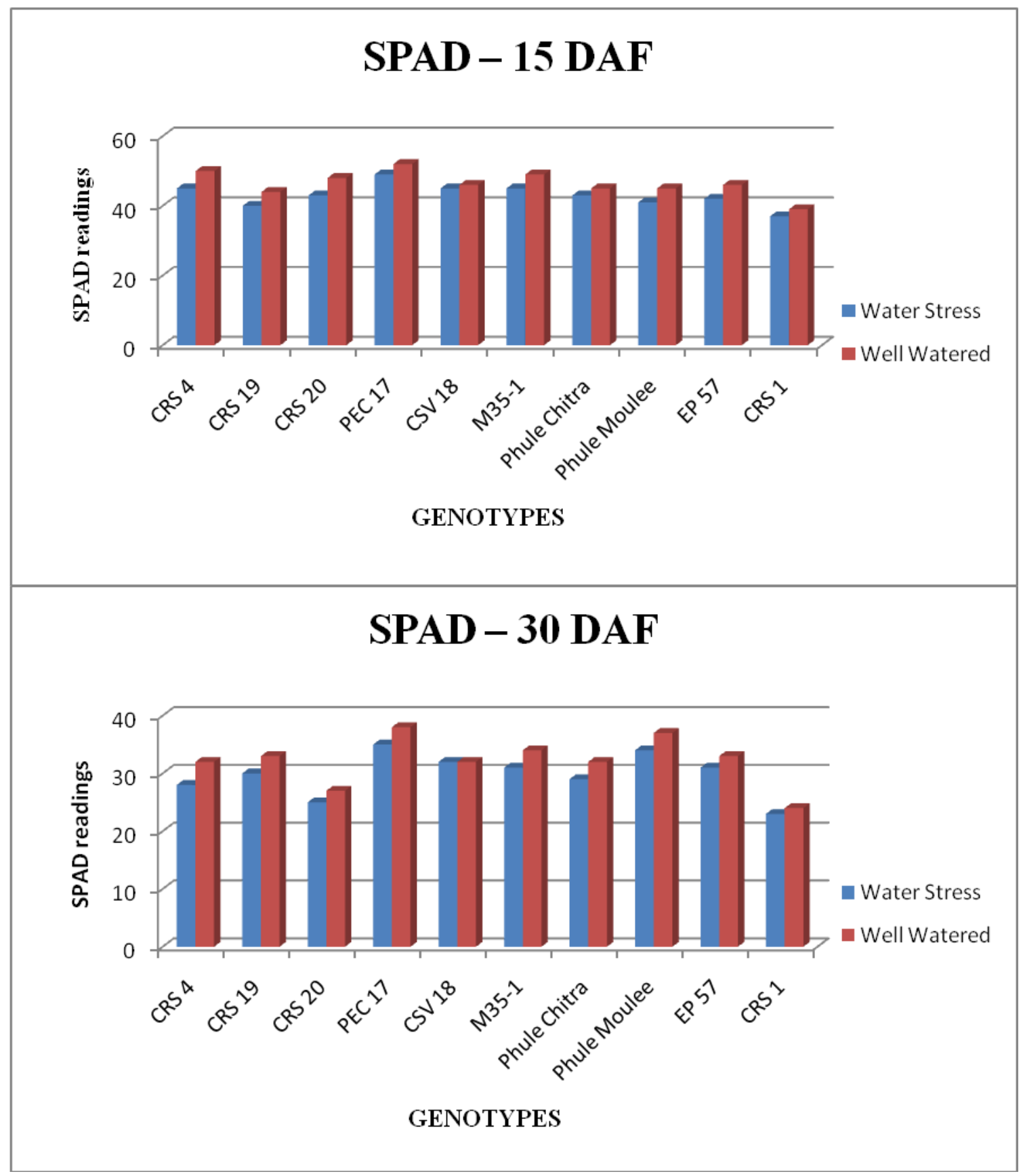


Fig.2 Photosynthetic rate $\left(\mu \mathrm{mol} \mathrm{CO} \mathrm{CO}^{2} \mathrm{~s}^{-1}\right)$ at $15 \mathrm{DAF}$ and $30 \mathrm{DAF}$ of Sorghum genotypes under well watered and water stress conditions

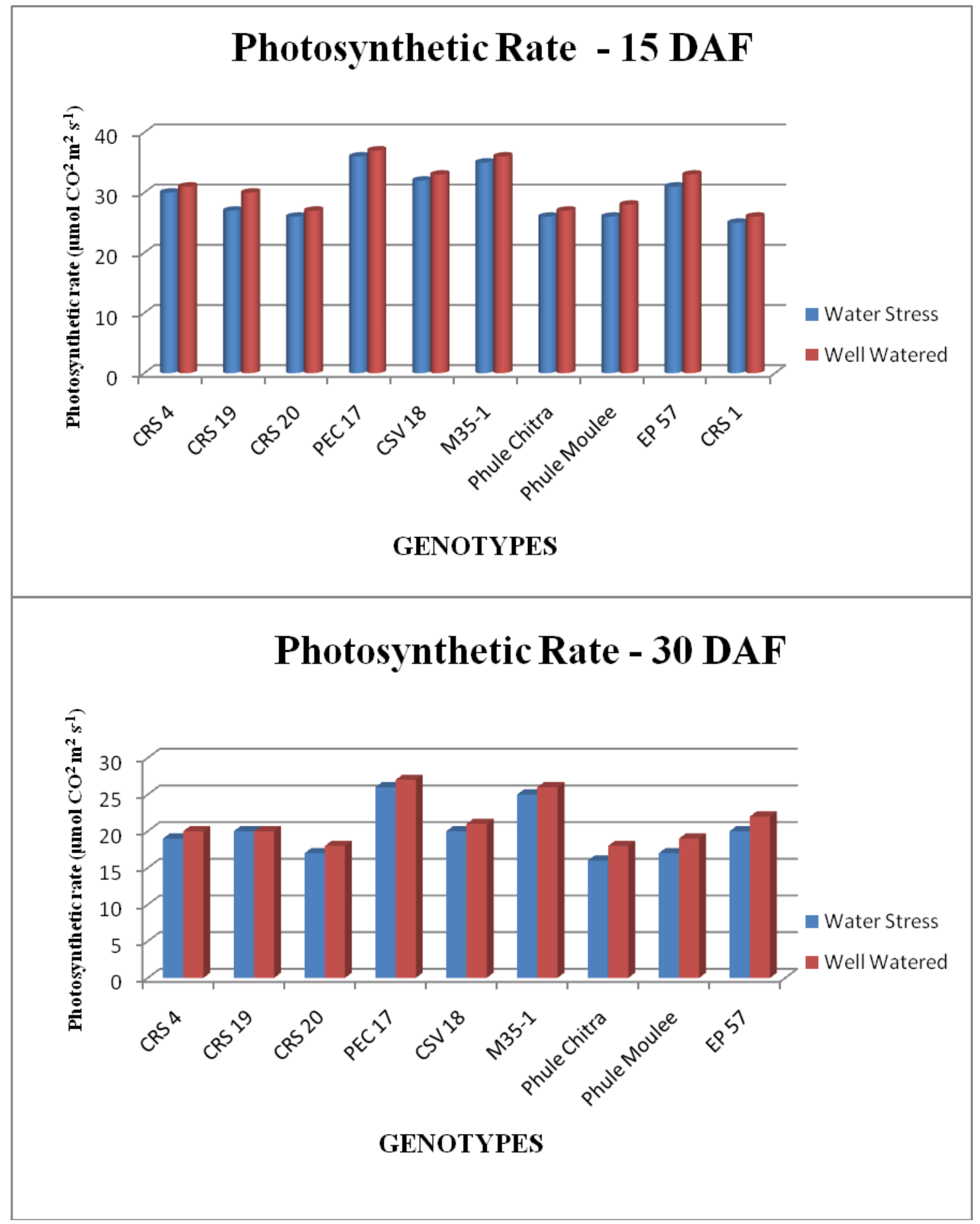


Fig.3 Transpiration rate $\left(\mu \mathrm{mol} \mathrm{H}_{2} \mathrm{O} \mathrm{m}^{2} \mathrm{~s}^{-1}\right)$ at $15 \mathrm{DAF}$ and 30 DAF of Sorghum genotypes as under well watered and water stress conditions

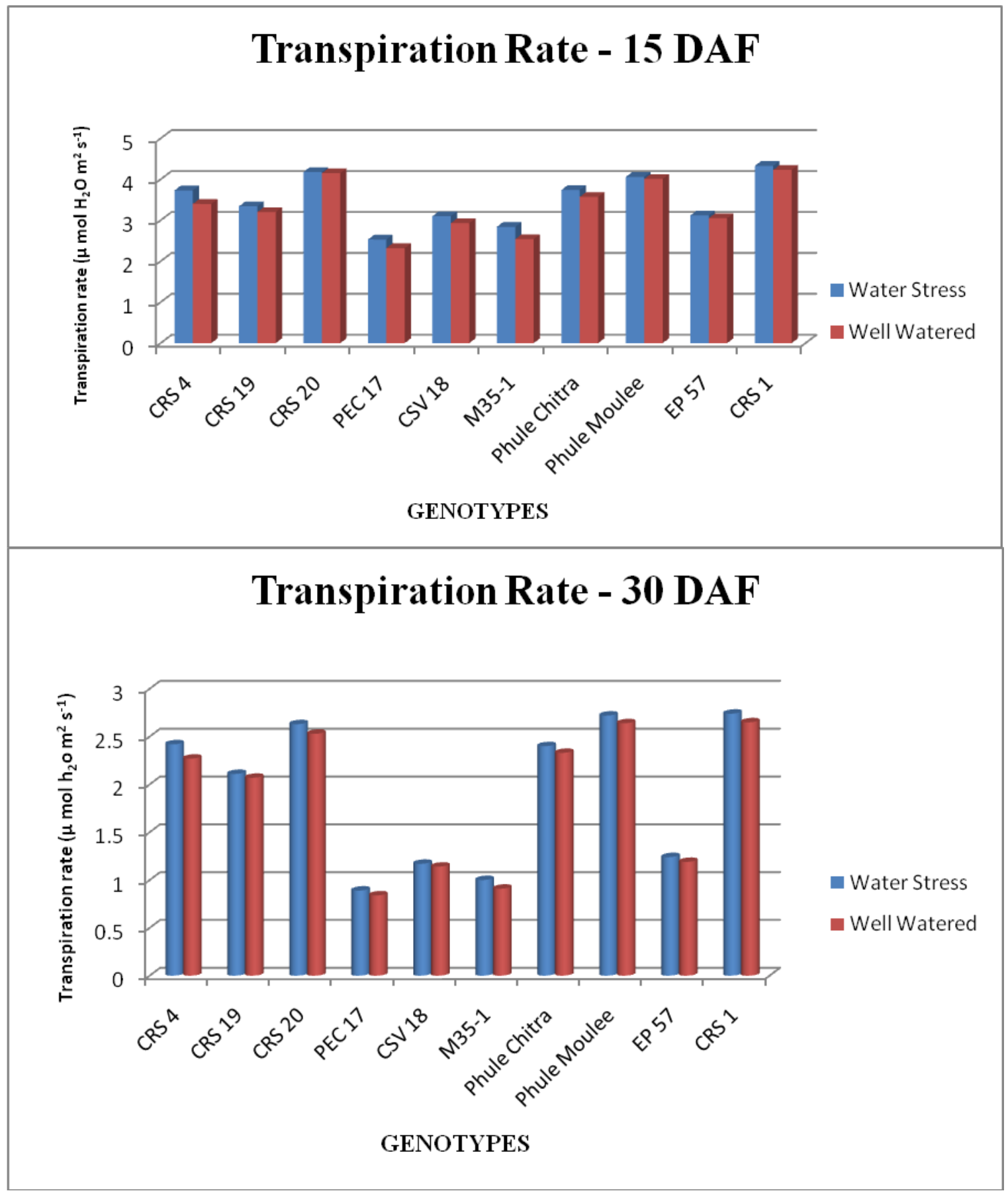


Fig.4 Stomata resistance $\left(\mathrm{s} . \mathrm{cm}^{-1}\right)$ at $15 \mathrm{DAF}$ and 30 DAF of Sorghum genotypes under well watered and water stress conditions

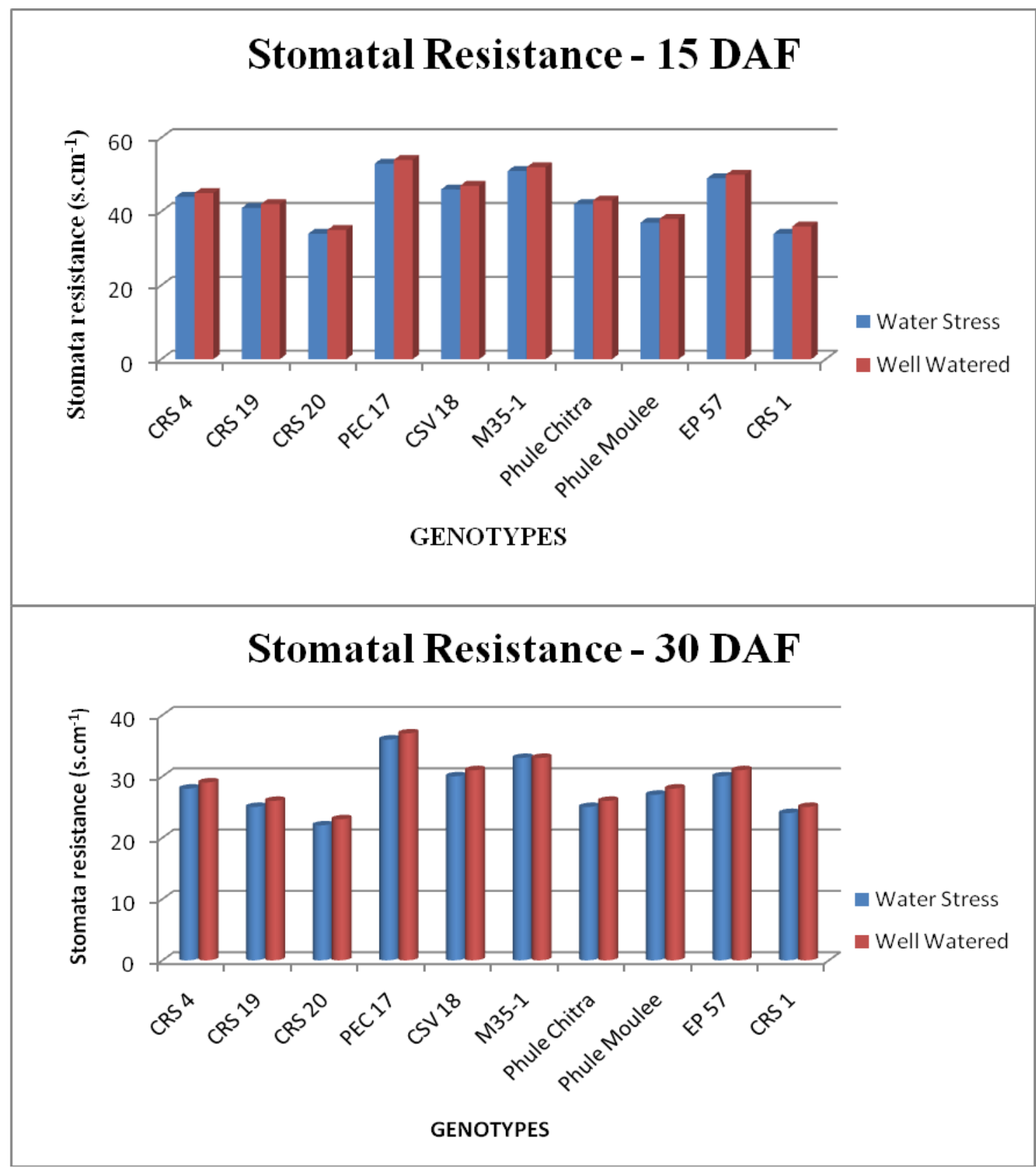

\section{Stomatal resistance $\left(\mathrm{s.cm}{ }^{-1}\right)$}

The data on stomatal resistance revealed significant differences among the genotypes both at 15 and 30 DAF and the maximum stomatal resistance was recorded at 15 DAF by all the genotypes compared to $30 \mathrm{DAF}$ (Table 4, Fig. 4).
At 15 DAF, the genotype PEC 17 had the maximum stomatal resistance followed by $\mathrm{M}$ 35-1 and EP 57. The lowest stomatal resistance was observed in CRS 1. At $30 \mathrm{DAF}$ also the maximum stomatal resistance was recorded by PEC 17 followed by M 35-1 and EP 57. The lowest stomatal resistance at this stage was recorded in the genotype CRS 1. 
The interaction between genotypes and stress treatments was significant and among the genotypes PEC 17 recorded highest stomatal resistance at $15 \mathrm{DAF}$ in well watered (54 $\left.\mathrm{s.cm}{ }^{-1}\right)$ and water stress (53 s.cm-1) conditions. The lowest stomatal resistance in well watered $\left(38 \mathrm{s.cm}^{-1}\right)$ and water stress (37 $\mathrm{s.cm}{ }^{-1}$ ) conditions was observed in the genotype CRS 1. Similar trend was observed at $30 \mathrm{DAF}$ with highest stomatal resistance in PEC 17 in well watered $\left(37 \mathrm{s.cm}^{-1}\right)$ and water stress $\left(36 \mathrm{s.cm}^{-1}\right)$ conditions. The lowest stomatal resistance in well watered $\left(25 \mathrm{s.cm}^{-1}\right)$ and water stress $\left(24 \mathrm{s.cm}^{-1}\right)$ conditions was observed in the genotype CRS 1 .

It was observed in our study that the transpiration rate decreased from $15 \mathrm{DAF}$ to 30 DAF in all the genotypes. In general, the genotypes which had maximum transpiration rate had low stomatal diffusive resistance. At $30 \mathrm{DAF}$, the maximum transpiration rate was observed in CRS 1 which also had the minimum stomatal diffusive resistance. While at 15 and $30 \mathrm{DAF}$, the genotypes PEC 17 also had low transpiration rate and considerably more stomatal diffusive resistance. This clearly indicates that these genotypes were able to maintain low leaf temperature which is a desirable character. These results are in accordance with the findings of Rao et al., (2001) and Pawar et al., (2005).

\section{References}

Ashok Surwenshi, V.P., Chimmad, R.L., Ravikumar. 2007. Comparative Studies of Hybrids and Parents for Physiological Parameters and Yield in Sorghum Karnataka Journal of Agricultural Sciences. 20 (1): 25 - 28.

Channappagoudar, B.B., Biradar, N.R., Bharamagoudar, T.D and Rokhade, J. 2008. Morpho-physiological Traits of Sorghum Parental Lines Determining Grain Yield and Biomass Karnataka
Journal of Agricultural Sciences. 21(2): 168-170.

Dhopte, A.M., Raghangadale, S.L and Jamadar, S.L. 1987. Physiological evaluation of forty exotic and wild sorghum lines in relation to stomatal factors involved in drought resistance. Annals of Plant Physiology, 1: 143 150.

Pawar, K.N., Biradar, B.D., Shamarao Jahagirdar, M.R and Ravikumar. 2005. Identification of Germplasm sources for adaptation under receding soil moisture situations in rabi Sorghum Agriculture Science Digest 25 (1): 56 - 58.

Rao, S.S., Seetharama, N., Kiran Kumar, K.A and Vanderlip, R.L. 2001. Characterization of sorghum growth stages NRCS Bulletin Series NO. 14. National Research Centre for Sorghum Rajendranagar. Hyderabad AP. pp: 115.

Rao, S.S., More, P.R., Solunke, V.D., kusalkar, D.V., Jirali, D.I., Pawar, K.N., Channappagoudar, B.B., Chimmad, V.P., Prabhakar and Rana, B.S. 2003. Physiological approaches for improving drought tolerance in rabi Sorghum. Proceedings of National Seminar on 'Role of Plant Physiology for Sustaining Quality and Quantity of Food Production in relation to Environment'. held at University of Agricultural Sciences. Dharwad. 26-32.

Sudhakar, P., Latha, P., Babitha, M., Prasanthi, L., Reddy, P.V. 2006. Physiological traits contributing to grain yields under drought in black gram and green gram Indian Journal of Plant Physiology.11 (4): 391-396.

Talwar, H.S., Prabhakar, M., Elangovan, Aruna, K., Rao, S.S., Mishra, J and Patil, V.J. 2011. Strategies to Improve Post flowering Drought Tolerance in Rabi Sorghum for Predicted Climate Change Scenario. Crop Improvement.37 
(2): 93-98.

Watling, J.R., Press, M.C. and Quick, W.P. 2003. Elevated $\mathrm{CO}_{2}$ induces biochemical and ultra structural changes in leaves of the $\mathrm{C}_{4}$ cereal Sorghum. Plant Physiology. 123(3): 1143-1152.

$\mathrm{Xu}, \mathrm{W} .$, Rosenow, D.T and Nguyen, H.T. 2000. Stay green trait in grain sorghum: relationship between visual rating and leaf chlorophyll concentration. PlantBreeding. 119(4): 365-367.

Yadav, S., Jyothi Lakshmi, N., Maheshwari, M. and Venkateswarlu, B. 1991. Influence of water deficit at vegetative, anthesis and grain fillings stages on water relation and grain yield in sorghum. Indian Journal of Plant Physiology. 10(1): 20-24.

\section{How to cite this article:}

Devkumar, D., V. Padma, H.S. Talwar and Farzana Jabeen. 2019. Study on Association of Biophysiological Parameters with Grain Yield in Sorghum Genotypes under Post Flowering Moisture Stress Conditions. Int.J.Curr.Microbiol.App.Sci. 8(02): 1601-1612. doi: https://doi.org/10.20546/ijcmas.2019.802.188 